\title{
First Trimester Mean Platelet Volume, Neutrophil to Lymphocyte Ratio, and Platelet to Lymphocyte Ratio Values Are Useful Markers for Predicting Preeclampsia
}

\author{
Süleyman Cemil Oğlak, Assoc Prof Dr, ${ }^{1}$ Şeyhmus Tunç, MD, ${ }^{1}$ Fatma Ölmez, MD ${ }^{2}$ \\ ${ }^{1}$ Department of Obstetrics and Gynecology, Health Sciences University, Gazi Yaşargil Training and Research Hospital, Diyarbakır, Turkey \\ ${ }^{2}$ Department of Obstetrics and Gynecology, Health Sciences University, Kanuni Sultan Süleyman Training and Research Hospital, Istanbul, \\ Turkey
}

Background: Preeclampsia complicates $2 \%$ to $8 \%$ of all pregnancies. Systemic inflammatory response (SIR) markers are widely used in the diagnosis of many inflammatory diseases and in the prediction of complicated pregnancies. This study examined the diagnostic value of SIR markers during the first trimester of pregnancy to predict preeclampsia development.

Methods: This retrospective case-control study was conducted from January 2020 to May 2020. We included 94 patients diagnosed with mild preeclampsia, 107 patients diagnosed with severe preeclampsia, and 100 normotensive pregnant patients as controls. We obtained the first trimester (6 to 14 weeks) complete blood cell counts for all patients. We used a receiver operating characteristic curve to evaluate the cutoff, sensitivity, and specificity values.

Results: First trimester mean platelet volume (MPV), neutrophil to lymphocyte ratio (NLR), and platelet to lymphocyte ratio (PLR) values were significantly higher in patients who developed preeclampsia in later pregnancy weeks. The optimal cutoff value for MPV was $10.65 \mathrm{fL}$, with a sensitivity of $63.7 \%$ and a specificity of $65.0 \%$. The best predictor for preeclampsia was NLR at an optimal cutoff value of 4.12 , with a sensitivity of $82.1 \%$ and specificity of $62.0 \%$. At a cutoff value of 131.8 , PLR predicted preeclampsia with a sensitivity rate of $65.0 \%$ and a specificity rate of $60.2 \%$.

Conclusion: The results of this study suggest that first trimester MPV, NLR, and PLR values are clinically useful markers in the prediction of preeclampsia. The increased first trimester values of MPV, NLR, and PLR also indicate that inflammation may play a crucial role in preeclampsia pathogenesis.

Keywords: Hypertension-pregnancy-induced, inflammation, preeclampsia, pregnancy, systemic inflammatory response

Address correspondence to Süleyman Cemil Oğlak, Assoc Prof Dr, Department of Obstetrics and Gynecology, Health Sciences University, Gazi Yaşargil Training and Research Hospital, Elazığ Road 10. km, Üçkuyular location 21070, Kayapınar, Diyarbakır, Turkey. Tel: +905064021157. Email: sampson_21@hotmail.com

\section{INTRODUCTION}

Preeclampsia is a severe syndrome marked by newonset hypertension after 20 weeks of gestation accompanied by proteinuria or various maternal end-organ dysfunctions, leading to maternal and fetal-neonatal morbidity and mortality. ${ }^{1}$ This hypertensive disorder complicates $2 \%$ to $8 \%$ of all pregnancies and is responsible for more than 70,000 maternal deaths and 500,000 fetal deaths worldwide every year. ${ }^{1,2}$ Preeclampsia is a systemic disorder that may cause multiple maternal organ dysfunctions, including renal, hepatic, pulmonary, neurologic, and hematologic complications. ${ }^{3,4}$ Also, preeclampsia may result in fetal complications, including oligohydramnios, growth restriction, preterm birth, placental abruption, and perinatal death. ${ }^{5,6}$

Preeclampsia is considered a 2-stage disorder; defective trophoblast invasion and spiral artery remodeling fail- ure are postulated as the primary step responsible for preeclampsia pathogenesis. ${ }^{1}$ Consequently, impaired uteroplacental blood flow results in hypoxia. The placenta's oxidative stress causes the release of factors, including proinflammatory cytokines, antiangiogenic agents, exosomes, and cell-free fetal DNA, into the maternal circulation that further trigger endothelial dysregulation and increased vascular permeability. ${ }^{7,8}$

Because of this pathophysiologic cascade, various biomarkers have attracted interest in the second decade of the 21 st century for their potential utility in preeclampsia prediction as early as the first trimester. ${ }^{9}$ Identifying patients with increased preeclampsia risk and predicting this disease early improve outcomes and enable prophylaxis with aspirin and early intervention. ${ }^{10}$ Studies have demonstrated that hyperactivation of inflammatory cells and immunologic 
responses of lymphocytes and neutrophils occur by releasing proinflammatory biomarkers driving endothelial dysregulation. ${ }^{11,12}$

The phrase low-grade inflammation is used to explain situations identified by a slightly increased immune cell count and elevated proinflammatory protein values in cases with no sign of an inflammatory disease. ${ }^{13}$ Systemic inflammatory response (SIR) markers, including red cell distribution width (RDW), plateletcrit (PCT), mean platelet volume (MPV), platelet distribution width (PDW), platelet to lymphocyte ratio (PLR), and neutrophil to lymphocyte ratio (NLR), are readily available as systemic inflammation markers from a complete blood count (CBC) and are widely used in the diagnosis of many inflammatory diseases and in the prediction of complicated pregnancies. ${ }^{11,14,15}$ However, published results regarding the association between preeclampsia and SIR markers remain controversial with conflicting reports. Whereas some studies demonstrated these markers to be useful in predicting preeclampsia, other studies did not. ${ }^{13,16}$

This study investigated the diagnostic value of SIR markers during the first trimester of pregnancy to predict preeclampsia development.

\section{METHODS}

This retrospective case-control study was conducted at the Department of Obstetrics and Gynecology at Diyarbakır Gazi Yaşargil Training and Research Hospital from January 2020 to May 2020. The ethics committee of the hospital approved the study (28.04.2020/462).

The study groups consisted of hospitalized and delivered mild or severe preeclamptic patients between 24 to 40 weeks of gestation. Control group patients were randomly selected from among healthy normotensive pregnant females without proteinuria, hospitalized for delivery at $\geq 37$ weeks of pregnancy during the same period. We matched the 3 groups for age and body mass index (BMI). The exclusion criteria for study participants were as follows: patients with inadequate data, multiple gestations, molar pregnancy, prepregnancy BMI $\geq 30 \mathrm{~kg} / \mathrm{m}^{2}$, a history of recurrent miscarriages or infertility, known thrombophilia or any other medical condition needing chronic drug treatment, previous pregnancy with gestational hypertensive disorders, complicated pregnancies (preterm delivery, preterm premature rupture of membranes, chorioamnionitis, intrauterine fetal demise, gestational diabetes mellitus, intrahepatic cholestasis of pregnancy), patients with malignancies, the use of acetylsalicylic acid and smoking during pregnancy, and fetuses with chromosomal or morphologic abnormalities.

During the study period, we identified 107 patients with mild preeclampsia, identified 116 patients with severe preeclampsia, and randomly selected 113 healthy pregnant controls. After applying the exclusion criteria and withholding patients with missing medical reports, 94 patients remained in the mild preeclampsia group, 107 patients remained in the severe preeclampsia group, and 100 patients remained in the control group.

We used the American College of Obstetricians and Gynecologists criteria for preeclampsia definition. ${ }^{17}$ We diagnosed mild preeclampsia after 20 weeks of gestation when systolic blood pressure (BP) was $\geq 140 / 90 \mathrm{mmHg}$ or diastolic BP was $\geq 90 \mathrm{mmHg}$ in at least 2 measurements made 4 hours apart in a patient with a previously normal BP and proteinuria ( $\geq 300 \mathrm{mg} / 24$-hour urine collection, protein to creatinine ratio of $\geq 0.3 \mathrm{mg} / \mathrm{g}$, or a dipstick reading of $2+$ protein). In the absence of proteinuria, patients with gestational hypertension were diagnosed with preeclampsia if they presented with any of the following severe features: thrombocytopenia (platelet count of $<100,000 / \mathrm{mL}$ ), renal insufficiency (serum creatinine level of $>1.1 \mathrm{mg} / \mathrm{dL}$ or a doubling of the serum creatinine concentration in the absence of renal disease), liver dysfunction (increased blood levels of transaminases to twice normal concentration), new-onset headache (unresponsive to medication and not accounted for by other diagnoses) or visual disturbances, and pulmonary edema.

We defined severe preeclampsia as systolic BP of $\geq 160$ $\mathrm{mmHg}$ or diastolic BP of $\geq 110 \mathrm{mmHg}$ on 2 occasions at least 4 hours apart in a patient with a previously normal BP and the presence of proteinuria ( $\geq 300 \mathrm{mg} / 24$-hour urine collection, protein to creatinine ratio of $\geq 0.3 \mathrm{mg} / \mathrm{g}$, or a dipstick reading of $2+$ protein). In the absence of proteinuria, patients with gestational hypertension were diagnosed with severe preeclampsia if they presented with any of the following severe features: thrombocytopenia, liver dysfunction, renal insufficiency, pulmonary edema, new-onset headache, or visual disturbances.

Age, gestational week, gravida, parity, and BMI were obtained by examining patients' medical records. BP data were obtained just prior to labor induction or cesarean section. Gestational week was examined by sonographic measurement and confirmed according to last menstrual period and first trimester ultrasound. We obtained the first trimester (6 to 14 weeks) CBC counts for all patients. When more than one $\mathrm{CBC}$ result was accessible, we recorded the closest result to 6 weeks of gestation for statistical analysis.

CBC values were measured with a Mindray BC 6800 (Mindray Bio-Medical Electronics $\mathrm{Co}$ ), an automatic blood counting device that uses laser and impedance measurement techniques. Hemoglobin, white blood cell count (WBC), neutrophil count, lymphocyte count, platelet count, RDW, MPV, PDW, and PCT values were extracted from patients' medical records. We calculated the NLR by dividing the neutrophil count by the lymphocyte count. We calculated the PLR by dividing the platelet count by the lymphocyte count.

IBM SPSS software, version 21.0 (IBM Corp) for Microsoft Windows was used for statistical evaluation of our research data. Measured variables are presented as mean $\pm S D$, and categorical variables are presented as numbers and percentages. We used the Kolmogorov-Smirnov test to determine if the numerical data matched the normality distribution. We used the $t$ test to compare the normally distributed data. Mann-Whitney $U$ test with Bonferroni correction and Kruskal-Wallis $\mathrm{H}$ test were used to compare the non-normally distributed data. We used the chi-square test to compare the qualitative variables. Differences were considered statistically significant at $P<0.05$. We used a receiver operating characteristic (ROC) curve to evaluate the cutoff, sensitivity, and specificity values. The cutoff value was estimated by using the Youden Index.

\section{RESULTS}

We included 94 patients diagnosed with mild preeclampsia, 107 patients diagnosed with severe preeclampsia, and 100 normotensive pregnant patients as controls. Patients' demographic characteristics and clinical features are 


\begin{tabular}{|c|c|c|c|c|c|c|}
\hline Variable & $\begin{array}{l}\text { Control } \\
\text { Group, } \\
n=100\end{array}$ & $\begin{array}{c}\text { Mild } \\
\text { Preeclampsia } \\
\text { Group, } n=94\end{array}$ & $\begin{array}{c}\text { Severe } \\
\text { Preeclampsia } \\
\text { Group, } n=107\end{array}$ & $\begin{array}{l}P_{1} \text { Value } \\
\text { (Control } \\
\text { vs Mild) }\end{array}$ & $\begin{array}{c}P_{2} \text { Value } \\
\text { (Mild vs } \\
\text { Severe) }\end{array}$ & $\begin{array}{c}P_{3} \text { Value } \\
\text { (Control } \\
\text { vs } \\
\text { Severe) }\end{array}$ \\
\hline Age, years & $27.4 \pm 6.1$ & $28.3 \pm 7.4$ & $28.7 \pm 6.8$ & 0.288 & 0.314 & 0.209 \\
\hline Gravidity & $2.79 \pm 1.69$ & $3.13 \pm 2.15$ & $3.24 \pm 2.07$ & 0.427 & 0.592 & 0.303 \\
\hline Parity & $1.59 \pm 1.57$ & $1.70 \pm 1.75$ & $1.88 \pm 1.99$ & 0.894 & 0.879 & 0.736 \\
\hline Weeks of gestation at the screening & $7.4 \pm 1.2$ & $7.2 \pm 1.1$ & $7.4 \pm 1.3$ & 0.553 & 0.492 & 0.861 \\
\hline Systolic blood pressure, $\mathrm{mmHg}$ & $102.1 \pm 9.2$ & $131.9 \pm 15.8$ & $164.2 \pm 15.9$ & $<0.001$ & $<0.001$ & $<0.001$ \\
\hline Diastolic blood pressure, $\mathrm{mmHg}$ & $62.7 \pm 6.9$ & $92.9 \pm 8.1$ & $112.1 \pm 10.0$ & $<0.001$ & $<0.001$ & $<0.001$ \\
\hline
\end{tabular}

Notes: Values (other than $P$ values) are mean \pm SD. Blood pressure data were obtained just prior to labor induction or cesarean section.

presented in Table 1. We found no significant differences among the 3 groups in terms of maternal age, gravidity, parity, and gestational week at the screening. The mean systolic and diastolic BPs were significantly higher in the mild and severe preeclampsia groups vs healthy controls.

First trimester SIR marker values of the participants are shown in Table 2. First trimester hemoglobin, RDW, lymphocyte count, PDW, and PCT values were comparable among all 3 groups. First trimester WBC counts were significantly higher in the mild and severe preeclampsia groups than in healthy controls $(P<0.001$ and $P<0.001$, respectively). First trimester neutrophil count was significantly lower in the control group than in the mild and severe preeclampsia groups ( $P=0.003$ and $P<0.001$, respectively). However, first trimester WBC and neutrophil counts were similar between the mild and severe preeclampsia groups. First trimester platelet counts were significantly higher in the mild and severe preeclampsia groups vs the control group ( $P=0.015$ and $P=0.004$, respectively). First trimester MPV value was significantly lower in healthy controls than in the mild and severe preeclampsia groups $(P<0.001$ and $P<0.001$, respectively). However, no significant difference was found regarding first trimester platelet count and MPV value between the mild and severe preeclampsia group ( $P=0.352$ and $P=0.505$, respectively).

First trimester NLR values were significantly higher in patients with mild and severe preeclampsia vs the control group $(P<0.001$ and $P<0.001$, respectively). Also, first trimester PLR values were significantly higher in patients with mild and severe preeclampsia than in healthy controls ( $P=0.016$ and $P=0.020$, respectively). Nonetheless, first trimester NLR and PLR values were similar between the mild and severe preeclampsia groups $(P=0.441$ and $P=0.988$, respectively).

We used ROC curves to derive cutoff values of MPV, NLR, and PLR to predict preeclampsia (Table 3). The optimal cutoff value for MPV was $10.65 \mathrm{fL}$, with a sensitivity of $63.7 \%$ and a specificity of $65.0 \%$ (Figure 1). The best predictor for preeclampsia was NLR at an optimal cutoff value of 4.12 , with a sensitivity of $82.1 \%$ and specificity of $62.0 \%$

Table 2. First Trimester Systemic Inflammatory Response Marker Values by Group

\begin{tabular}{|c|c|c|c|c|c|c|}
\hline Variable & $\begin{array}{l}\text { Control } \\
\text { Group, } \\
n=100\end{array}$ & $\begin{array}{c}\text { Mild } \\
\text { Preeclampsia } \\
\text { Group, } n=94\end{array}$ & $\begin{array}{c}\text { Severe } \\
\text { Preeclampsia } \\
\text { Group, } n=107\end{array}$ & $\begin{array}{l}P_{1} \text { Value } \\
\text { (Control } \\
\text { vs Mild) }\end{array}$ & $\begin{array}{l}P_{2} \text { Value } \\
\text { (Mild vs } \\
\text { Severe) }\end{array}$ & $\begin{array}{c}P_{3} \text { Value } \\
\text { (Control } \\
\text { vs } \\
\text { Severe) }\end{array}$ \\
\hline Hemoglobin, g/dL & $11.7 \pm 1.4$ & $12.2 \pm 1.5$ & $11.9 \pm 1.7$ & 0.226 & 0.280 & 0.392 \\
\hline Red cell distribution width, $\%$ & $15.2 \pm 2.3$ & $14.5 \pm 1.7$ & $14.9 \pm 2.3$ & 0.074 & 0.066 & 0.117 \\
\hline White blood cell count, $\mathrm{mm}^{3} \times 10^{3}$ & $9.7 \pm 1.7$ & $11.2 \pm 2.9$ & $11.6 \pm 2.9$ & $<0.001$ & 0.243 & $<0.001$ \\
\hline Neutrophil count, $\times 10^{3} / \mu \mathrm{L}$ & $6.8 \pm 1.7$ & $8.2 \pm 3.1$ & $8.6 \pm 3.5$ & 0.003 & 0.235 & $<0.001$ \\
\hline Lymphocyte count, $\times 10^{3} / \mu \mathrm{L}$ & $2.0 \pm 0.5$ & $2.0 \pm 0.6$ & $2.1 \pm 0.8$ & 0.863 & 0.691 & 0.735 \\
\hline Platelet count, $\mathrm{mm}^{3} \times 10^{3}$ & $242.5 \pm 57.5$ & $259.7 \pm 48.5$ & $266.7 \pm 70.6$ & 0.015 & 0.352 & 0.004 \\
\hline Platelet distribution width, $\%$ & $16.2 \pm 1.4$ & $16.5 \pm 0.3$ & $16.4 \pm 0.3$ & 0.079 & 0.126 & 0.099 \\
\hline Mean platelet volume, $\mathrm{fL}$ & $10.3 \pm 1.3$ & $10.6 \pm 2.3$ & $11.2 \pm 1.4$ & $<0.001$ & 0.505 & $<0.001$ \\
\hline Plateletcrit, \% & $0.2 \pm 0.06$ & $0.2 \pm 0.06$ & $0.2 \pm 0.07$ & 0.429 & 0.463 & 0.386 \\
\hline Neutrophil to lymphocyte ratio & $3.1 \pm 1.1$ & $4.3 \pm 1.4$ & $4.6 \pm 1.8$ & $<0.001$ & 0.441 & $<0.001$ \\
\hline Platelet to lymphocyte ratio & $121.1 \pm 27.4$ & $137.1 \pm 44.9$ & $138.1 \pm 38.2$ & 0.016 & 0.988 & 0.020 \\
\hline
\end{tabular}

Note: Values (other than $P$ values) are mean \pm SD. 
Table 3. Area Under the Receiver Operating Characteristic (ROC) Curve of Mean Platelet Volume (MPV), Neutrophil to Lymphocyte Ratio (NLR), and Platelet to Lymphocyte Ratio (PLR)

\begin{tabular}{cccccc}
\hline & & & \multicolumn{2}{c}{$\mathbf{9 5 \%} \mathbf{C l}$} & \\
\cline { 4 - 5 } Variable & ROC & Standard Error & Lower & Upper & P Value \\
\hline MPV & 0.663 & 0.033 & 0.598 & 0.728 & $<\mathbf{0 . 0 0 1}$ \\
NLR & 0.767 & 0.030 & 0.709 & 0.826 & $<\mathbf{0 . 0 0 1}$ \\
PLR & 0.631 & 0.034 & 0.565 & 0.698 & $<\mathbf{0 . 0 0 1}$ \\
\hline
\end{tabular}

(Figure 2). At a cutoff value of 131.8, PLR predicted preeclampsia with a sensitivity rate of $65.0 \%$ and a specificity rate of $60.2 \%$ (Figure 3 ).

\section{DISCUSSION}

The current study demonstrated that first trimester MPV, NLR, and PLR values were significantly higher in patients who developed preeclampsia in later pregnancy weeks. However, these markers were not predictive in discriminating the severe preeclampsia cases from the mild cases.

Chronic inflammation has a central role in the pathogenesis of preeclampsia. During a healthy pregnancy, the balance between $\mathrm{T}$ helper 1 (Th1) and Th2 immune cells and their immune responses is crucial for maintaining gestation. ${ }^{18,19} \mathrm{~A}$ healthy pregnancy is thought to be a state with Th2 predominance, which provides an immunotolerant setting for fetal rejection prevention. ${ }^{20}$ However, in preeclamptic patients, placental ischemia as a result of shallow trophoblast invasion increases proinflammatory CD4+ T cells and reduces regulatory $\mathrm{T}$ helper cells. ${ }^{19}$ Th1/Th2 balance is disrupted, and a shift toward the Th1 response causes a chronic proinflammatory state with Th1 predominance. ${ }^{21}$ The ischemic placenta produces cytokines, including interleukin-6, tumor necrosis factor alpha, and soluble fms-like tyrosine kinase

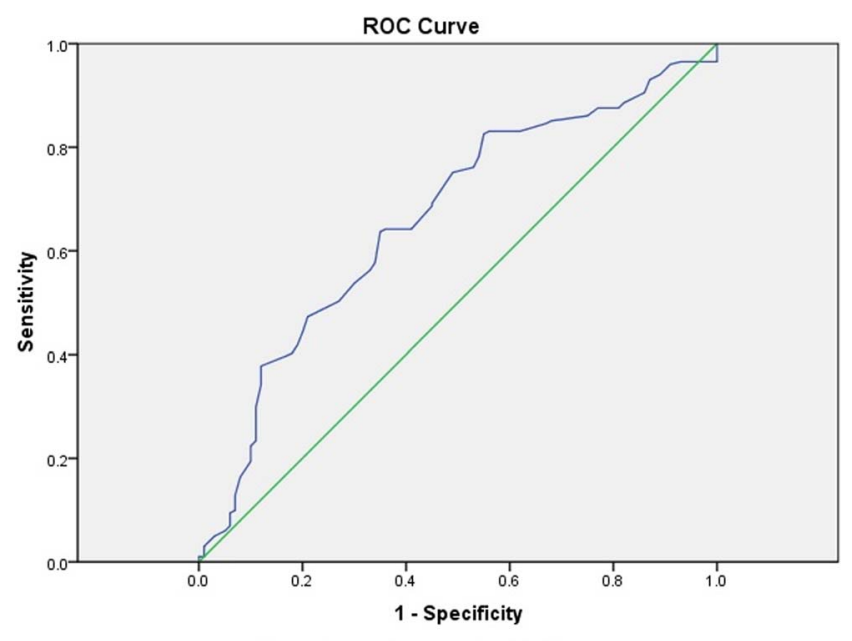

Diagonal segments are produced by ties.

Figure 1. Receiver operating characteristic (ROC) curve for first trimester mean platelet volume value for predicting preeclampsia. The area under the curve is $0.663(95 \% \mathrm{Cl}$ 0.598-0.728).

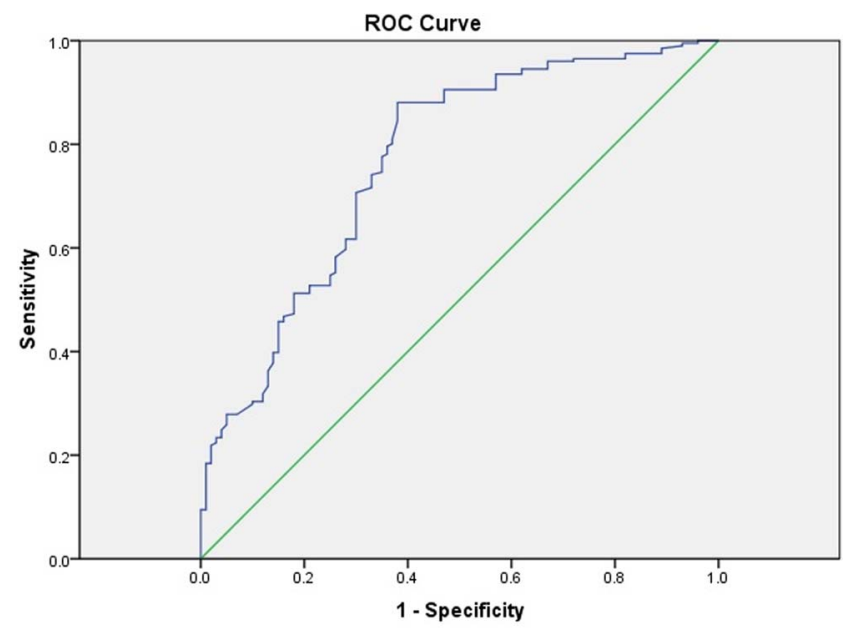

Diagonal segments are produced by ties.

Figure 2. Receiver operating characteristic (ROC) curve for first trimester neutrophil to lymphocyte ratio value for predicting preeclampsia. The area under the curve is 0.767 (95\% Cl 0.709-0.826).

1 (sFlt-1), and stimulates peripheral $\mathrm{T}$ cell activation that contributes to hypertension development by inducing $B$ cells responsible for agonistic angiotensin II type 1 and endothelin-1 receptor autoantibodies. ${ }^{22}$ Understanding the mechanism underlying this pathophysiologic stage supports research about screening tests determining oxidative stress, endothelial dysfunction, and inflammatory markers for preeclampsia prediction. ${ }^{23} \mathrm{C}$-reactive protein, fibrinogen, alpha-1 antitrypsin, angiotensinogen, interleukins, alpha1-acid glycoprotein, and ceruloplasmin are the acute phase inflammatory proteins that are elevated in patients with preeclampsia. ${ }^{24}$ Also, Akolekar et al reported that screening by the combination of maternal characteristics, mean arterial

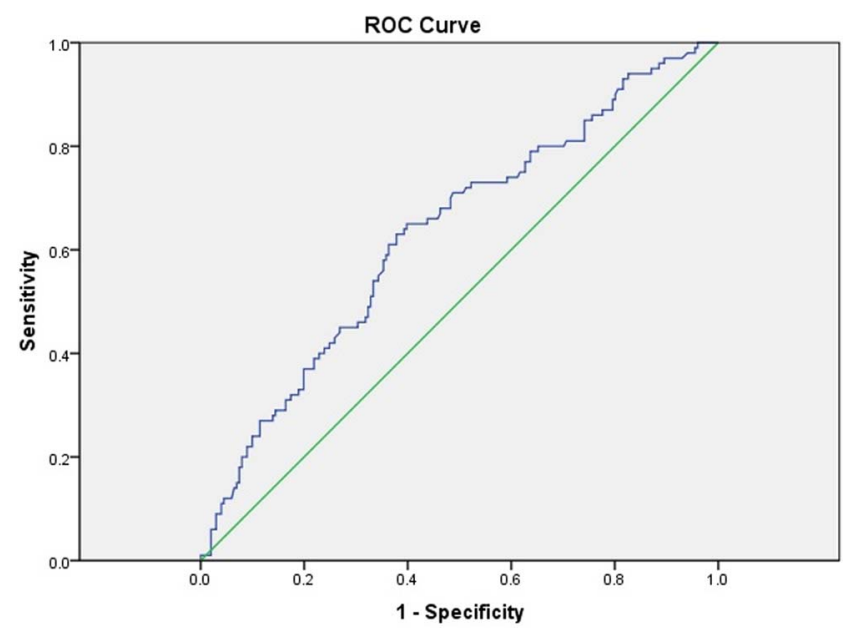

Diagonal segments are produced by ties.

Figure 3. Receiver operating characteristic (ROC) curve for first trimester platelet to lymphocyte ratio value for predicting preeclampsia. The area under the curve is $0.631(95 \%$ Cl 0.565-0.698). 
pressure, uterine artery pulsatility index, serum pregnancyassociated plasma protein $\mathrm{A}$, and placental growth factor detected $54 \%$ of all cases of preeclampsia, $<40 \%$ of cases of term preeclampsia, and $96 \%$ of cases of preeclampsia requiring delivery before 34 weeks of gestation. ${ }^{25}$ In their study, Rolnik et $\mathrm{al}^{26}$ included pregnant patients at high risk for preterm preeclampsia following the Akolekar et al criteria. Rolnik and colleagues indicated that administration of lowdose acetylsalicylic acid to these patients reduced the risk of preterm preeclampsia but did not reduce the incidence of term preeclampsia. ${ }^{26}$ Therefore, an effective method for predicting preeclampsia in the first trimester does not yet exist.

The diagnostic value of SIR markers such as NLR, PLR, PDW, MPV, PCT, and RDW in many diseases such as coronary artery disease, autoimmune diseases, inflammatory diseases, and malignancies has already been shown. ${ }^{27,28}$ However, the few studies focusing on preeclampsia have conflicting results on the association between the value of these markers in the first trimester and increased risk of preeclampsia development. ${ }^{13,16}$ We planned our study to evaluate whether these markers, which can be quickly obtained from the $\mathrm{CBC}$, changed in patients before preeclampsia.

High RDW values are thought to reflect increased inflammation and oxidative stress, but the mechanism has not been fully established. ${ }^{29}$ Sen-Yu et al examined RDW values in the second trimester and reported that RDW values were higher in pregnant patients who developed preeclampsia in the third trimester. ${ }^{30}$ However, Çintesun et al stated that RDW levels did not differ between preeclamptic patients and healthy pregnant patients. ${ }^{31}$ We found no association between first trimester RDW values and subsequent preeclampsia development in our study.

In addition to their primary function in hemostasis, platelets are efficient immune modulators and effectors. ${ }^{32}$ Endothelial dysfunction induces vasoconstriction and platelet adhesion and aggregation, triggering coagulation and resulting in hypoxic damage to the endothelium. ${ }^{33}$ Thrombocyte consumption in the maternal peripheral circulation stimulates bone marrow production. The younger platelets produced at this stage are larger than the older ones and show a strong tendency toward aggregation. ${ }^{34}$ Therefore, the number, volume, and function of platelets change, and platelet turnover demonstrates an increase in preeclamptic maternal vasculature. ${ }^{34,35}$ Platelet count, PDW, MPV, and PCT are regarded as platelet activation markers. ${ }^{36}$ Tzur and Sheiner and Gezer et al reported high first trimester platelet counts in patients who develop preeclampsia. ${ }^{37,38}$ Kirbas et al found that while platelet count, MPV, and PCT values were similar among patients in the first trimester, PDW values were higher in the patients who later developed preeclampsia. ${ }^{13}$ Mannaerts et al stated that among platelet markers, only MPV was significantly increased before the 20th week of pregnancy in patients who subsequently developed preeclampsia vs healthy individuals. ${ }^{39}$ In our study, first trimester platelet count and MPV values were significantly higher in patients who developed preeclampsia than in the control group. This result suggests that platelet activation may have a crucial role in the pathogenesis of inflammation in preeclampsia. However, PDW and PCT values were comparable between the groups.
The interaction between platelets and vascular endothelium induces the release of inflammatory substances (chemokines, adhesion proteins, growth factors), and these substances cause leukocyte migration and adhesion. ${ }^{40}$ Canzoneri et al reported that the total leukocyte count was significantly increased in preeclamptic patients vs healthy pregnant females. ${ }^{41}$ The increased total leukocyte count was primarily attributable to the rise in neutrophil count. Our study found that WBC count and neutrophil count were significantly higher in patients who developed preeclampsia in the later weeks of gestation vs the controls. Kirbas et al demonstrated that first trimester NLR values were significantly higher in patients who subsequently developed preeclampsia vs healthy pregnant patients, and PLR values were significantly higher in patients who developed severe preeclampsia vs the mild preeclampsia group and the control group. ${ }^{13}$ Moreover, Kirbas et al reported that pregnant patients with high first trimester NLR values were significantly more likely to develop severe preeclampsia. Mannaerts et al reported that first trimester NLR and PLR values were similar in patients who would develop preeclampsia and healthy individuals. ${ }^{39}$ Gezer et al indicated that high PLR and NLR values during the first trimester are objective predictors of preeclampsia and could be used for early preeclampsia diagnosis. ${ }^{38}$ Likewise, our study demonstrated that first trimester NLR and PLR values were significantly higher in patients with established preeclampsia. However, these values were not useful in discriminating patients with severe preeclampsia from those with mild cases. Therefore, we suggest that enhanced inflammation and platelet activation already exist in the early gestation weeks, and first trimester NLR and PLR values might serve as a predictor of preeclampsia.

This study's major strength is that we examined first trimester CBC results to predict subsequent preeclampsia, parameters evaluated by a minority of previous research. Also, we stratified the patients with preeclampsia into mild and severe groups.

A limitation of this study is its retrospective design, with the limitations of such studies. Also, the change in NLR and PLR values during the course of the disease is unknown. Another limitation is the absence of levels of proinflammatory cytokines, including sFlt-1 and soluble endoglin, that have been previously identified with preeclampsia. A study that correlates the results of SIR markers with these cytokines may provide more insight into preeclampsia prediction.

\section{CONCLUSION}

The results of this study suggest that first trimester MPV, $N L R$, and PLR values are clinically useful markers in the prediction of preeclampsia. The increased first trimester values of MPV, NLR, and PLR also indicate that inflammation may play a crucial role in preeclampsia pathogenesis.

\section{ACKNOWLEDGMENTS}

The authors have no financial or proprietary interest in the subject matter of this article.

\section{REFERENCES}

1. Rana S, Lemoine E, Granger JP, Karumanchi SA. Preeclampsia: pathophysiology, challenges, and perspectives. Circ Res. 2019;124(7):1094-1112. doi: 10.1161/CIRCRESAHA.118.313276 
2. Duley L. The global impact of pre-eclampsia and eclampsia. Semin Perinatol. 2009;33(3):130-137. doi: 10.1053/j.semperi.2009.02.010

3. Tomimatsu T, Mimura K, Matsuzaki S, Endo M, Kumasawa K, Kimura T. Preeclampsia: maternal systemic vascular disorder caused by generalized endothelial dysfunction due to placental antiangiogenic factors. Int $\mathrm{J} \mathrm{Mol} \mathrm{Sci.}$ 2019;20(17):4246. doi: 10.3390/ijms20174246

4. Oğlak SC, Tunç Ş, Obut M, Şeker E, Behram M, Tahaoğlu AE. Maternal near-miss patients and maternal mortality cases in a Turkish tertiary referral hospital. Ginekol Pol. 2021;92(4):300-305. doi: 10.5603/GP.a2020.0187

5. Magro-Malosso ER, Saccone G, Di Tommaso M, Roman A, Berghella V. Exercise during pregnancy and risk of gestational hypertensive disorders: a systematic review and meta-analysis. Acta Obstet Gynecol Scand. 2017;96(8):921-931. doi: 10.1111/aogs.13151

6. Oğlak SC, Bademkıran MH, Obut M. Predictor variables in the success of slow-release dinoprostone used for cervical ripening in intrauterine growth restriction pregnancies. J Gynecol Obstet Hum Reprod. 2020;49(6):101739. doi: 10.1016/j.jogoh.2020.101739

7. Burton GJ, Redman CW, Roberts JM, Moffett A. Pre-eclampsia: pathophysiology and clinical implications. BMJ. 2019;366:I2381. doi: 10.1136/bmj.I2381

8. Obut M, Oğlak SC. Expression of CD44 and IL-10 in normotensive and preeclamptic placental tissue. Ginekol Pol. 2020;91(6):334-341. doi: 10.5603/GP.2020.0058

9. Pihl K, Sørensen S, Stener Jørgensen F. Prediction of preeclampsia in nulliparous women according to first trimester maternal factors and serum markers. Fetal Diagn Ther. 2020;47(4):277-283. doi: 10.1159/000503229

10. Chaiworapongsa T, Chaemsaithong P, Korzeniewski SJ, Yeo L, Romero R. Pre-eclampsia part 2: prediction, prevention and management. Nat Rev Nephrol. 2014;10(9):531-540. doi: 10.1038/nrneph.2014.103

11. Oğlak SC, Aydın MF. Are neutrophil to lymphocyte ratio and platelet to lymphocyte ratio clinically useful for the prediction of early pregnancy loss? Ginekol Pol. 2020;91(9):524-527. doi: 10.5603/GP.a2020.0082

12. Laresgoiti-Servitje E, Gómez-López N, Olson DM. An immunological insight into the origins of pre-eclampsia. Hum Reprod Update. 2010;16(5):510-524. doi: 10.1093/humupd/dmq007

13. Kirbas A, Ersoy AO, Daglar K, et al. Prediction of preeclampsia by first trimester combined test and simple complete blood count parameters. J Clin Diagn Res. 2015;9(11):QC20-3. doi: 10.7860/JCDR/2015/15397.6833

14. Tang J, Gao X, Zhi M, et al. Plateletcrit: a sensitive biomarker for evaluating disease activity in Crohn's disease with low hs-CRP. J Dig Dis. 2015;16(3):118-124. doi: 10.1111/1751-2980.12225

15. Wang J, Zhu QW, Cheng XY, et al. Assessment efficacy of neutrophil-lymphocyte ratio and monocyte-lymphocyte ratio in preeclampsia. J Reprod Immunol. 2019;132:29-34. doi: 10.1016/j.jri.2019.02.001

16. Kashanian M, Hajjaran M, Khatami E, Sheikhansari N. Evaluation of the value of the first and third trimester maternal mean platelet volume (MPV) for prediction of pre-eclampsia. Pregnancy Hypertens. 2013;3(4):222-226. doi: 10.1016/j.preghy.2013.06.001

17. ACOG practice bulletin no. 202: gestational hypertension and preeclampsia. Obstet Gynecol. 2019;133(1):1. doi: 10.1097/AOG.0000000000003018
18. Behram M, Oğlak SC. The expression of angiogenic protein Cyr61 significantly increases in the urine of early-onset preeclampsia patients. J Contemp Med. 2021;11(5): 605-609. doi: $10.16899 / \mathrm{jcm} .945345$

19. Harmon AC, Cornelius DC, Amaral LM, et al. The role of inflammation in the pathology of preeclampsia. Clin Sci (Lond). 2016;130(6):409-419. doi: 10.1042/CS20150702

20. Perez-Sepulveda A, Torres MJ, Khoury M, Illanes SE. Innate immune system and preeclampsia. Front Immunol. 2014;5:244. doi: 10.3389/fimmu.2014.00244

21. Amaral LM, Wallace K, Owens M, LaMarca B. Pathophysiology and current clinical management of preeclampsia. Curr Hypertens Rep. 2017;19(8):61. doi: 10.1007/s11906-017-0757-7

22. Spradley FT, Palei AC, Granger JP. Immune mechanisms linking obesity and preeclampsia. Biomolecules. 2015;5(4):3142-3176. doi: 10.3390/biom5043142

23. Behram M, Oğlak SC, Doğan Y. Evaluation of BRD4 levels in patients with early-onset preeclampsia. J Gynecol Obstet Hum Reprod. 2021;50(2):101963. doi: 10.1016/j.jogoh.2020.101963

24. McCarthy FP, Ryan RM, Chappell LC. Prospective biomarkers in preterm preeclampsia: a review. Pregnancy Hypertens. 2018;14:72-78. doi: 10.1016/j.preghy.2018.03.010

25. Akolekar R, Syngelaki A, Poon L, Wright D, Nicolaides KH. Competing risks model in early screening for preeclampsia by biophysical and biochemical markers. Fetal Diagn Ther. 2013;33(1):8-15. doi: 10.1159/000341264

26. Rolnik DL, Wright D, Poon LC, et al. Aspirin versus placebo in pregnancies at high risk for preterm preeclampsia. N Engl J Med. 2017;377(7):613-622. doi: 10.1056/NEJMoa1704559

27. Seckin KD, Karslı MF, Yucel B, et al. The utility of tumor markers and neutrophil lymphocyte ratio in patients with an intraoperative diagnosis of mucinous borderline ovarian tumor. Eur J Obstet Gynecol Reprod Biol. 2016;196:60-63. doi: 10.1016/j.ejogrb.2015.10.025

28. Wada H, Dohi T, Miyauchi K, et al. Mean platelet volume and long-term cardiovascular outcomes in patients with stable coronary artery disease. Atherosclerosis. 2018;277:108-112. doi: 10.1016/j.atherosclerosis.2018.08.048

29. Li N, Zhou H, Tang Q. Red blood cell distribution width: a novel predictive indicator for cardiovascular and cerebrovascular diseases. Dis Markers. 2017;2017:7089493. doi: 10.1155/2017/7089493

30. Sen-Yu W, Chao X. Assessment of the relationship between red blood cell distribution width and preganecy hypertension disease. J Obstet Gynaecol Res. 2016;42(10):1258-1262. doi: $10.1111 /$ jog. 13067

31. Çintesun E, Incesu Çintesun FN, Ezveci H, Akyürek F, Çelik Ç. Systemic inflammatory response markers in preeclampsia. $J$ Lab Physicians. 2018;10(3):316-319. doi: 10.4103/JLP.JLP_144_17

32. Jenne $C N$, Kubes P. Platelets in inflammation and infection. Platelets. 2015;26(4):286-292. doi: 10.3109/09537104.2015.1010441

33. Alijotas-Reig J, Palacio-Garcia C, Farran-Codina I, Ruiz-Romance M, Llurba E, Vilardell-Tarres M. Circulating cell-derived microparticles in severe preeclampsia and in fetal growth restriction. Am J Reprod Immunol. 2012;67(2):140-151. doi: 10.1111/j.1600-0897.2011.01072.x

34. Juan P, Stefano G, Antonella S, Albana C. Platelets in pregnancy. J Prenat Med. 2011;5(4):90-92.

35. Kim MA, Han GH, Kwon JY, Kim YH. Clinical significance of platelet-to-lymphocyte ratio in women with preeclampsia. Am J Reprod Immunol. 2018;80(1):e12973. doi: 10.1111/aji.12973 
36. Karateke A, Kurt RK, Baloğlu A. Relation of platelet distribution width (PDW) and platelet crit to preeclampsia. Ginekol Pol. 2015;86(5):372-375. doi: 10.17772/gp/2425

37. Tzur T, Sheiner E. Is there an association between platelet count during the first trimester and preeclampsia or other obstetric complications later in pregnancy? Hypertens Pregnancy. 2013;32(1):74-82. doi: 10.3109/10641955.2012.704109

38. Gezer C, Ekin A, Ertas IE, et al. High first-trimester neutrophil-to-lymphocyte and platelet-to-lymphocyte ratios are indicators for early diagnosis of preeclampsia. Ginekol Pol. 2016;87(6):431-435. doi: 10.5603/GP.2016.0021

39. Mannaerts D, Heyvaert S, De Cordt C, Macken C, Loos C, Jacquemyn Y. Are neutrophil/lymphocyte ratio (NLR), platelet/lymphocyte ratio (PLR), and/or mean platelet volume (MPV) clinically useful as predictive parameters for preeclampsia? J Matern Fetal Neonatal Med. 2019;32(9):1412-1419. doi: 10.1080/14767058.2017.1410701

40. Bakogiannis C, Sachse M, Stamatelopoulos K, Stellos K. Platelet-derived chemokines in inflammation and atherosclerosis. Cytokine. 2019;122:154157. doi: 10.1016/j.cyto.2017.09.013

41. Canzoneri BJ, Lewis DF, Groome L, Wang Y. Increased neutrophil numbers account for leukocytosis in women with preeclampsia. Am J Perinatol. 2009;26(10):729-732. doi: $10.1055 / \mathrm{s}-0029-1223285$

This article meets the Accreditation Council for Graduate Medical Education and the American Board of Medical Specialties Maintenance of Certification competencies for Patient Care and Medical Knowledge.

(C2021 by the author(s); licensee Ochsner Journal, Ochsner Clinic Foundation, New Orleans, LA. This article is an open (c) (i) access article distributed under the terms and conditions of the Creative Commons Attribution (CC BY) license (creativecommons.org/licenses/by/4.0/legalcode) that permits unrestricted use, distribution, and reproduction in any medium, provided the original author(s) and source are credited. 